\title{
Optical phase lock loop as high-Q filter for optical frequency comb lines selection
}

\author{
K. Balakier *, H. Shams, M. J. Fice, L. Ponnampalam, C. S. Graham, C. C. Renaud and A. J. Seeds \\ Department of Electronic and Electrical Engineering, \\ University College London (UCL), \\ Torrington Place, London WC1E 7JE, United Kingdom \\ *k.balakier@ucl.ac.uk
}

\begin{abstract}
This paper describes the first optical phase lock loop (OPLL) based on a photonic integrated circuit (PIC) fabricated using the generic foundry process and off-the-shelf electronic components. The PIC contains a DBR laser that can be frequency and phase stabilized in reference to the external laser or, what is demonstrated for the first time in this paper, in respect to Optical Frequency Comb Generator (OFCG) line. The OFCG offers 19 highly coherent lines spaced by 15 GHz. The OPLL can attenuate all adjacent channels and noise down to -60 $\mathrm{dBm}$ resulting in only a single optical line being present at the output. The OPLL output is a single mode DBR laser offset from the reference optical tone by 4-12 GHz. Hence, OPLL can be considered as ultra-selective optical filter. The laser can by tunead by current across $1 \mathrm{THz}(8 \mathrm{~nm})$ range to match the wavelength of the reference tone. The coherence between DBR laser and comb lines is demonstrated by measurements of the heterodyne signal residual phase noise level which is below $\mathbf{- 1 0 0}$ $\mathrm{dBc} / \mathrm{Hz}$ at $5 \mathrm{kHz}$ offsets from the carrier. This is a record low value for the OPLL based on monolithically integrated photonic circuit. Such an OPLL could be used for high purity, tuneable millimetre and $\mathrm{THz}$ waves generation.
\end{abstract}

Keywords- optical phase lock loop; THz signal generation; photonic integration; tuneable millimetre-wave source component;

\section{INTRODUCTION}

Optical Phase Lock Loop (OPLL) based on semiconductor diode lasers has been investigated for several years [1], [2], [3], [4] as it can offer advantages in terms of spectral characteristics and wavelength stability typical for highquality external cavity lasers, but in the footprint and at the cost of a simple semiconductor laser. Additionally, the heterodyne OPLL allows to stabilize the slave laser in respect to the master laser with a tuneable, yet precisely defined frequency offset between these two lasers. In the process of phase locking the slave laser acquires the spectral characteristics of the master laser. This may be advantageous in applications where, for instance, spectral characteristics of ultra-stable master laser can be transferred onto a number of inexpensive diode lasers [5]. Other applications in which OPLL can be utilized include coherent communication, coherent LIDAR or millimetre and $\mathrm{THz}$ waves generation [6]. For the latter applications it is of particular interest to stabilize the OPLL while using optical frequency comb generator (OFCG) as reference. In this arrangement the OPLL could be considered as an ultra-selective optical "filter" with an optical gain.

The OPLL presented in this paper was designed in such a way that none of the optical input signals is present at the OPLL output, which offers the suppression of noise and the other comb lines down to the level of $-60 \mathrm{dBm}$ in the close proximity from the centre wavelength, as the OPLL does not have a Gaussian shape which is typical for optical filters. Moreover, the output of the OPLL can be higher that the power of the individual comb line. Hence, the OPLL subsystem may also offer an optical gain, which is a great asset when compared to conventional passive optical filters.

Realising the OPLL based on semiconductor laser presents a particular challenge due its rather broad linewidth. A feedback cycle must occur in less than a couple of nanoseconds time in order to successfully stabilize the laser with $\mathrm{MHz}$ range linewidth. Such a short delay cannot be achieved when using optical fibre one metre of fibre contributes approximately $5 \mathrm{~ns}$ delay. This explains the need for the photonic integration.

The OPLL presented in this work is based on the PIC fabricated using a generic foundry approach. The foundry allows for several different designs to be allocated and processed on a single wafer using generic fabrication process. This leads to a significant reduction in the fabrication cost, reduced lead time and increased yield comparing to custom fabrication processes. The drawback of foundry fabrication comes, similarly as its advantage, from generic and universal nature of the fabrication process, which may compromise performance of some photonic devices. Nevertheless, the presented OPLL makes a very good example of a system, where the foundry fabricated PIC and COTS electronic feedback loop were used to build an opto-electronic feedback loop capable of suppressing the phase noise of the heterodyne signal to the level never achieved before by monolithically integrated OPLL. The RMS jitter of the heterodyne signal generated by the lasers is less than $6.5 \mathrm{ps}$ and the residual phase noise is suppressed to the exceptionally low level of -100 $\mathrm{dBc} / \mathrm{Hz}$ at $5 \mathrm{kHz}$ offset from the carrier frequency.

\section{OPTICAL PHASE LOCK LOOP}

When the OPLL is in operation, two lasers are heterodyned on the photodiode resulting in the RF signal being generated. This heterodyne signal is then compared with the RF reference signal on the phase detector, and the baseband phase error signal, which corresponds to the phase difference between the 
lasers, is produced and fed back into the slave laser to correct its operation point in accordance with the master laser phase and frequency changes.

The experimental assembly used to phase stabilized the DBR laser to the incoming reference optical tone (single laser or optical comb line) is presented schematically in Fig. 1.

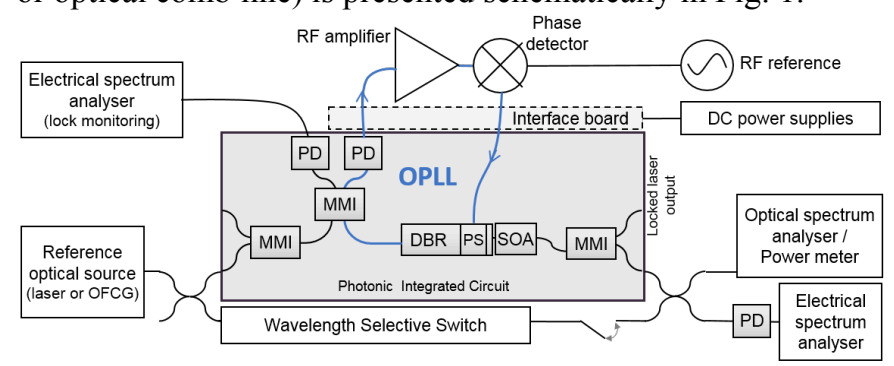

Fig. 1 Schematic of the experimental arrangement

\section{A. Photonic integrated circuit}

The key component of the OPLL system is an InP-based PIC consisting of DBR lasers, semiconductor amplifiers (SOA), PIN photodiodes (PIN-PD) and various optical interconnections. The PIC dimensions are $2 \mathrm{~mm} \times 6 \mathrm{~mm}$, and the layout design is presented in Fig. 2

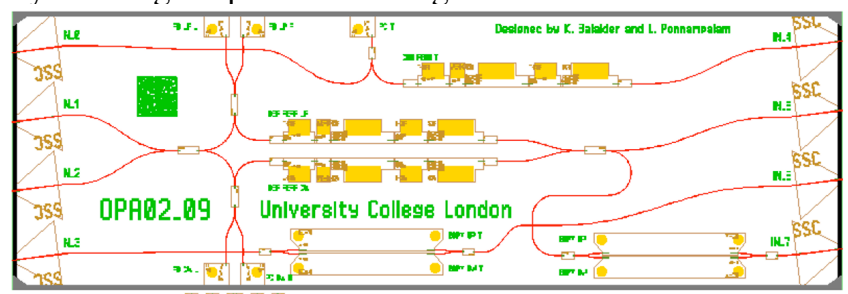

Fig. 2 PIC layout (This figure is going to be improved)

The laser integrated on the PIC consists of the multiquantum well gain section and two Bragg grating section creating lasers cavity. The laser wavelength can be tuned across $8 \mathrm{~nm}$ by adjusting current applied to the laser gratings. Moreover the laser has a short phase section (PS) which enables very fine tuning of the laser across $50 \mathrm{GHz}$ with the tuning sensitivity of up to $45 \mathrm{GHz} / \mathrm{mA}$ (depending on the phase section bias point). This phase section is used to adjust the laser operation point when the OPLL is in operation. The laser operates as a single mode with the side-mode suppression ration greater than $45 \mathrm{~dB}$. When the loop is in operation, the laser wavelength can be controlled with the Hz-level precision by adjusting the frequency of the RF reference synthesizer.

The side-illuminated PIN photodiodes are integrated on the PIC in such a way so that two PDs can measure the same heterodyne signal. One PIN-PD forms part of the feedback loop, while the other is used to control the quality of the lock (lock monitoring). The PIN-PDs have responsivity of $0.8 \mathrm{~A} / \mathrm{W}$, and the frequency response was measured to decrease by $3 \mathrm{~dB}$ at $2 \mathrm{GHz}$ and by $6 \mathrm{~dB}$ at $12 \mathrm{GHz}$.

The PIC also contains passive components used to guide the signal on the PIC, such as single mode waveguides, multimode interference (MMI) couples/splitters, bends, tapers and converters. To ensure the most efficient fibre to/from chip coupling, the waveguide spot size converters were included at the edge of the chip. Moreover, to reduce back reflections the waveguides at the edge of the PIC were positioned at an angle and antireflective coating was applied. During the experiments the reference optical signal was coupled to the PIC using lensed fiber, while a simple cleaved single mode fibre was used at the PIC output. Up to $0 \mathrm{dBm}$ optical power was measured at the output fibre when the DBR laser gain section and the SOA were driven with $100 \mathrm{~mA}$ and $50 \mathrm{~mA}$ currents, respectively.

\section{B. Electronic feedback circuit and OPLL performance}

The electronic part of the feedback loop consists of commercial off-the-shelf (COTS) components, including bias tees to provide negative bias to PIC-PDs, RF amplifier, doublebalanced mixer and laser phase section bias circuit, which also has a function of low-pass loop filter. The total delay within the loop is $1.7 \mathrm{~ns}$ and the loop is realized as first order type I loop.

The interface PCB has been implemented between the PIC on carrier and electronic components to provide electrical connections and match the dimensions difference between the electrical pads designed for wirebonding on the alumina boards next to PIC and SMA connectors of the COTS components. The OPLL requires neither DC nor RF probes for its operation, and the presented semi-packaged solution makes the OPLL portable, hence easily implementable as a part of bigger systems. The picture of the OPLL assembly is presented in Fig. 3.

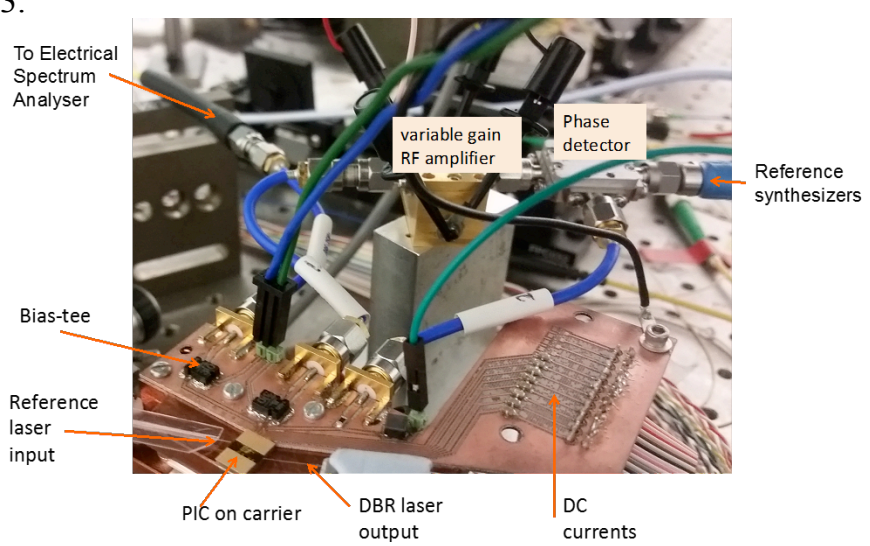

Fig. 3 Photograph of the OPLL assembly (this figure is going to be improved)

Both RF amplifier and phase detector are also available as the surface mount components, hence could potentially be implemented on a single PCB together with bias-tee and biasing circuitry, which in turn would reduce the OPLL size as well as phase error propagation delay, resulting in the increase of the loop bandwidth.

The double-balanced mixer is used in the OPLL as a multiplier-type phase detector, which produces baseband error signal from the product of the heterodyne and the reference RF waveforms. This "analog nature" of the phase detector allows for changes of phase detector gain and, in consequence, loop gain by changing the amplitude of one of the input signals. The RF power level at the input to the phase detector can be variable and precisely adjusted by the variable gain of the RF amplifier. These changes, according to the OPLL theory [7], have an effect on the OPLL bandwidth which can be observed and measured on the electrical spectra of the heterodyne signal, as demonstrated in Fig. 4. 


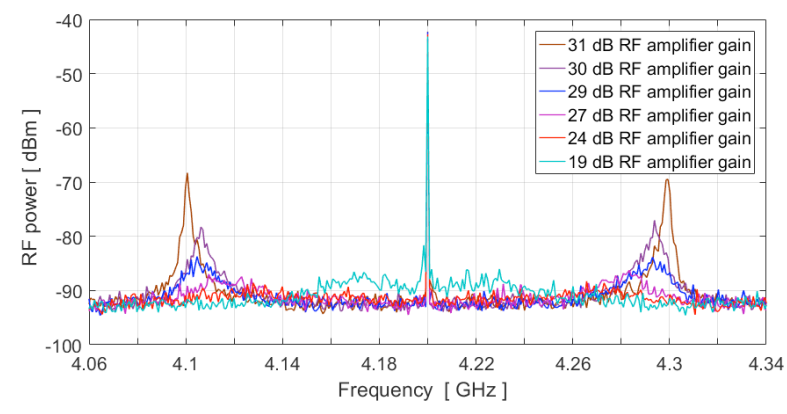

Fig. 4 Electrical spectra of the phase-locked heterodyne measured at different $\mathrm{RF}$ amplifier gains

Fig. 4 demonstrates how changes in the RF amplifier gain from $19 \mathrm{~dB}$ to $31 \mathrm{~dB}$ results in the change of the loop bandwidth from approximately $20 \mathrm{MHz}$ to $100 \mathrm{MHz}$, respectively. Also, as the loop gain increases, the phase noise close to the carrier frequency is suppressed more and eventually the secondary peak power rises. Further increase of the gain will result in appearance of additional peaks on both sides of the carrier, which indicates unstable locking, and an eventual loss of lock.

Our approach to use analog type phase detector is very different from OPLLs with phase detectors based on sequential devices which built from logic gates that operate with binary rectangular input waveforms, and are characterised by fixed gain/loss [3], [8].

The OPLL allows the DBR laser to be phase stabilized to the reference optical tone with any frequency offset ranging from $4 \mathrm{GHz}$ to $12 \mathrm{GHz}$, as shown in Fig. 5. This could be further improved by replacing the phase detector, currently specified for 4-12 GHz operation range, with another one with brother operational bandwidth. The other component limiting the higher offset frequency locking is the PIN-PD, whose frequency response decreases by $6 \mathrm{~dB}$ at $12 \mathrm{GHz}$. The roll-off in the frequency response of both the photodiode and other electrical connections can be noticed on Fig. 5, where the power of $12 \mathrm{GHz}$ heterodyne is smaller than that of the $6 \mathrm{GHz}$ tone.

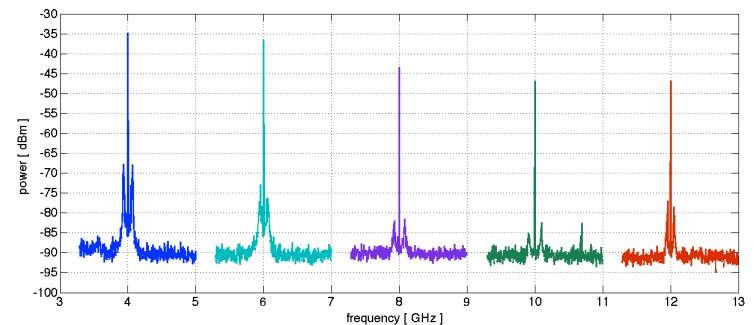

Fig. 5 Heterodyne beat-notes between integrated DBR lasers and external reference laser measured at the output of integrated PIN-PD. (this figure is going to be improved)

However, big frequency offset between the two lasers may not constitute a major requirement for many applications. For instance, when the OPLL is meant to be used to select one of the multiple lines of the OFCG, the highest frequency offset does not need to be greater than a half of the frequency spacing between the comb lines [7]. It is worth reminding that many combs have their lines separated by a dozen or so $\mathrm{GHz}$, which is often limited by the frequency bandwidth of the modulator and the driving synthesizer.

\section{LOCKING To OptiCAL FREQUENCY COMB GENERATOR LiNE}

For this experiment the single mode reference laser, in the experimental assembly presented in Fig. 1, was replaced by OFCG. The optical spectra of the comb at the input to the OPLL and consequently the single optical line of the phaselocked DBR laser at the output of the OPLL are presented in Fig. 6.

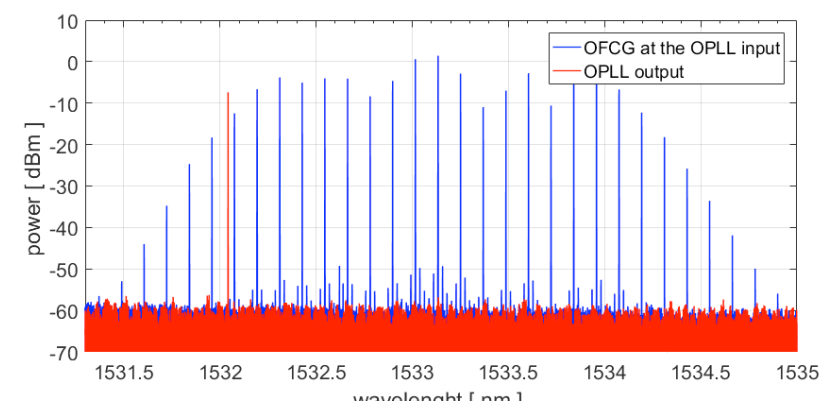

Fig. 6 Optical spectra of the optical frequency comb at the OPLL input and DBR laser at the OPLL output (this is not the final figure)

The optical frequency comb has a span of $285 \mathrm{GHz}$, within the $-15 \mathrm{~dB}$ envelope, and free spectral range of $15 \mathrm{GHz}$. It was generated using dual-drive MZM modulator [ ] followed by the EDFA to boost the power of each optical line. The peak power of the line used as OPLL reference was $-13 \mathrm{dBm}$. The laser used as reference for the OFCG has $10 \mathrm{kHz}$ FWHM linewidth and operates at $1533.13 \mathrm{~nm}$ wavelength. The entire comb with a total optical power of $\sim 0 \mathrm{dBm}$ was coupled into the lensed fibre and consequently into the waveguide on the PIC, before it reached the active area of photodetectors. This resulted in up to $-1 \mathrm{~mA}$ of DC photocurrent (depending on the photodiode revers bias) being generated at each PIN-PD. The output of the monitoring PIN-PD was connected to an electrical spectra analyser (ESA) so that several beat-notes resulting from heterodyning the integrated DBR laser output with the closest comb lines could be observed, as can be seen in Fig. 7.

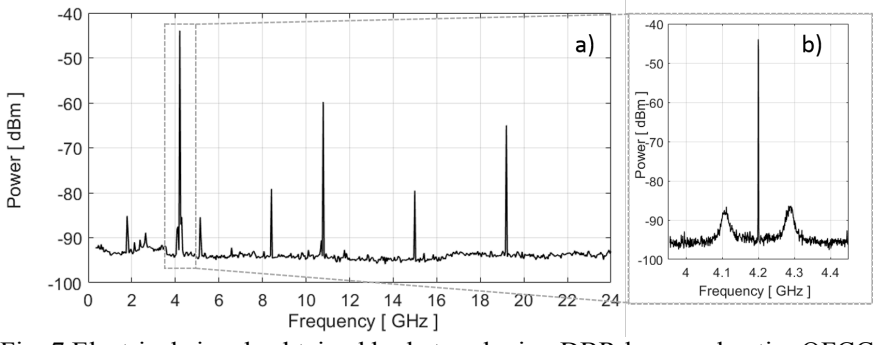

Fig. 7 Electrical signals obtained by heterodyning DBR laser and entire OFCG on the monitoring PIN-PD integrated in the PIC $(\mathrm{RBW}=\mathrm{VBW}=)$

Several tones can be identified in Fig. 7, including DBR beating with three closest comb lines resulting in $4.2 \mathrm{GHz}$ (included as an insert in Figure 1), $10.8 \mathrm{GHz}$ and $19.2 \mathrm{GHz}$ signals. The $15 \mathrm{GHz}$ beat between the comb lines is also visible. Lastly, the second harmonic of $4.2 \mathrm{GHz}$ heterodyne signal is present at $8.4 \mathrm{GHz}$ demonstrating the photomixing characteristics of the PIC-PD. Despite the multiple signals detected, the OPLL could successfully stabilise the DBR laser at the frequency offset defined by the RF synthesizer.

In order to further investigate the quality and stability of the locking, the optical frequency comb was split in two optical paths, as shown schematically in Fig. 1. To avoid an 
increased phase difference between two tones propagated through different length optical paths, an additional 5(TBC) $\mathrm{m}$ of optical fibre length was added to one of them. The OPLL was used to select one line of the comb, while a configurable wavelength selective switch with XX(TBC) nm FWHM bandwidth was used to isolate the next consecutive line. The two paths were then combined together on an external photodiode and the heterodyne signal was observed at the ESA demonstrating the high purity $34.3 \mathrm{GHz}$ signal $(30 \mathrm{GHz}$ spacing between two comb lines and $4.3 \mathrm{GHz}$ frequency offset introduced by OPLL) as seen in Fig. 8. The difference in the spectral purity of the heterodyne signal generated when the OPLL operated in the locked and unlocked conditions is clearly visible. The frequency of the phase-locked heterodyne can be tuned with Hz-level precision by simply adjusting the frequency of the RF reference inside the OPLL.

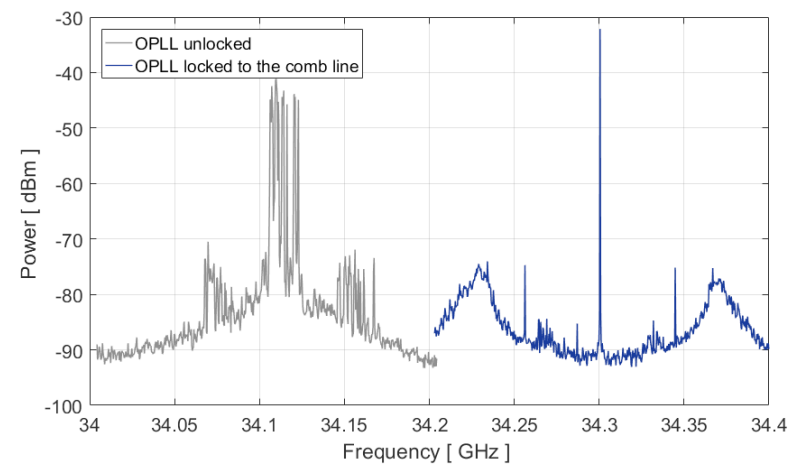

Fig. 8 Electrical spectra of the free running and phase locked heterodyne signals measured at the external photodiode $(\mathrm{RBW}=50 \mathrm{kHz}, \mathrm{VBW}=3 \mathrm{kHz})$

Subsequently, the phase noise of heterodyne signals was measured to assess the coherence between the comb line used as optical reference for the OPLL and the phase-stabilised DBR laser. Fig. 9 shows the phase noise measurements of the $4 \mathrm{GHz}$ and $34 \mathrm{GHz}$ heterodyne signals measured on the integrated PIC-PD and external photodiode, respectively. The beat-note between two comb lines separated by $30 \mathrm{GHz}$ is also included for the reference.

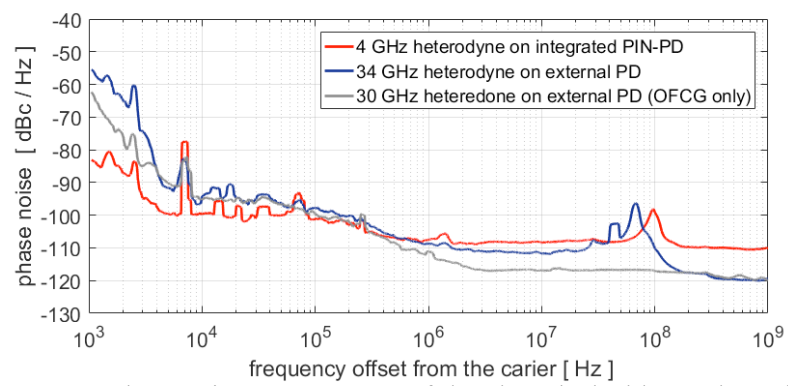

Fig. 9 SSB phase noise measurement of the phase locked heterodyne signals at $4 \mathrm{GHz}$ and $34 \mathrm{GHz}$

Fig. 9 demonstrates good agreement between spectral characteristics of the beat-note generated by two comb lines and those generated by one comb line and DBR laser. The OPLL can supress the phase noise of the heterodyne signal down to $-100 \mathrm{dBc} / \mathrm{Hz}$ at offsets from the carrier greater than 6 $\mathrm{kHz}$. The increase in the phase noise level at the lower frequency offsets can be explained by not exact length matching between the two optical paths through which the optical tones propagate. The increased phase noise level of the
$4 \mathrm{GHz}$ signal at the higher offset is due to the lower power signal measured by the integrated PIN-PD.

\section{CONCLUSIONS}

The optical phase lock loop based on the generic foundry photonic integrated circuit has been successfully phase locked to optical frequency comb for the first time. The potential of the OPLL to be used as high-quality filter for the optical frequency comb line selection is demonstrated. The OPLL input and output are optically isolated, which allows reducing the noise and adjacent lines to below $-60 \mathrm{dBc}$ power level, even in a close proximity to the centre wavelength. Importantly, integrated DBR laser generates more power than required by the loop for successful phase locking, hence the optical gain is offered. The heterodyne OPLL enables signal tuneability by shifting the output wavelength by $4-12 \mathrm{GHz}$ in respect to the input. The coherence between the OPLL input and output signals is assessed by measuring the spectral purity of the heterodyne signal, whose residual phase noise is supressed to below $-100 \mathrm{dBc} / \mathrm{Hz}$ at $5 \mathrm{kHz}$ offset from the carrier.

\section{ACKNOWLEDGMENT}

This work has been supported by the United Kingdom Engineering and Physical Sciences Research Council (EPSRC) through COTS project (EP/J017671/1) and European Commission Seventh Framework Programme (FP7-ICT) through IPHOBAC-NG (619870) and PARADIGM (257210) projects.

\section{REFERENCES}

[1] L. H. Enloe and J. L. Rodda, "Laser phase-locked loop," Proc. IEEE, vol. 53, no. 2, pp. 165-166, 1964.

[2] M. Kourogi, C.-H. Shin, and M. Ohtsu, "A $134 \mathrm{MHz}$ bandwidth homodyne optical phase-locked-loop of semiconductor laser diodes," Photonics Technol. Lett., vol. 3, no. 3, pp. 270-272, Mar. 1991.

[3] M. Lu, H. Park, E. Bloch, A. Sivananthan, A. Bhardwaj, Z. Griffith, L. A. Johansson, M. J. Rodwell, and L. A. Coldren, "Highly integrated optical heterodyne phase-locked loop with phase / frequency detection," Opt. Express, vol. 20, no. 9, pp. 1090-1092, 2012.

[4] K. Balakier, L. Ponnampalam, M. J. Fice, C. C. Renaud, and A. J. Seeds, "Integrated Semiconductor Laser Optical Phase Lock Loops," J. Sel. Top. Quantum Electron., vol. accepted f, 2017.

[5] N. Satyan, W. Liang, A. Yariv, and L. Fellow, "Coherence Cloning Using Semiconductor Laser Optical Phase-Lock Loops," J. Quantum Electron., vol. 45, no. 7, pp. 755-761, 2009.

[6] A. J. Seeds, M. J. Fice, K. Balakier, M. Natrella, O. Mitrofanov, M. Lamponi, M. Chtioui, F. van Dijk, M. Pepper, G. Aeppli, a. G. Davies, P. Dean, E. Linfield, and C. C. Renaud, "Coherent terahertz photonics," Opt. Express, vol. 21, no. 19, pp. 22988-23000, Sep. 2013.

[7] K. Balakier, M. J. Fice, L. Ponnampalam, A. J. Seeds, and C. C. Renaud, "Monolithically integrated optical phase lock loop for microwave photonics," J. Light. Technol., vol. 32, no. 20, pp. 38933900, Oct. 2014.

[8] R. J. Steed, F. Pozzi, M. Fice, C. Renaud, D. C. Rogers, I. F. Lealman, D. G. Moodie, P. J. Cannard, C. Lynch, L. Johnston, M. J. Robertson, R. Cronin, L. Pavlovic, L. Naglic, M. Vidmar, and A. J. Seeds, "Monolithically integrated heterodyne optical phase-lock loop with RF XOR phase detector," Opt. Express, vol. 19, no. 21, pp. 20048-53, Oct. 2011. 